\title{
Numerical Methods for 3D Tokamak Simulations using a Flux- surface independent Grid
}

\author{
Andreas Stegmeir ${ }^{1, *}$, David Coster ${ }^{1}$, Omar Maj ${ }^{1,}$, and Karl Lackner ${ }^{1,}$ \\ ${ }^{1}$ Max-Planck-Institut für Plasmaphysik, EURATOM Association, 85748 Garching, Germany \\ Received XXXX, revised XXXX, accepted XXXX \\ Published online XXXX
}

Key words Plasma Turbulence, X-point, Support Operator Method, Numerical Diffusion.

\begin{abstract}
A numerical approach for 3D Tokamak simulations using a flux surface independent grid is presented. The grid consists of few poloidal planes with a Cartesian Isotropic grid within each poloidal plane. Perpendicular operators can be discretised within a poloidal plane using standard second order finite difference methods. The discretisation of parallel operators is achieved with a field line following map and an interpolation. The application of the support operator method to the parallel diffusion operator conserves the self-adjointness of the operator on the discrete level and keeps the numerical decay rate at a low level. The developed numerical methods can be applied to geometries where an X-point is present.
\end{abstract}

Copyright line will be provided by the publisher

\section{Introduction}

Turbulent cross field transport of heat and particles in the scrape-off layer (SOL) of Tokamaks is to a large extent still an open issue [1,2]. The presence of many temporal-spatial scales simultaneously makes turbulence in the SOL more complex in contrast to core turbulence and requires a sophisticated set of equations. The geometry in presence of an X-point constitutes another complexity. Turbulent structures tend to have long wave lengths $(\sim m)$ along the magnetic field line and very small $(\sim m m)$ perpendicular structures. This property is usually exploited by aligning the coordinate system and the numerical grid to the magnetic field. However, at the separatrix the safety factor $q$ approaches infinity, which makes the use of such field aligned coordinate systems impossible [3]. In this paper a numerical approach is presented which can cope with geometries where an X-point is present. The main emphasis is on the discretisation of the parallel diffusion operator. In section 2 the construction of the numerical grid and the discretisation of the operators is presented. In section 3 the numerical convergence properties are discussed. The main result is that the numerical decay can be kept at very low levels, by maintaining the self-adjointness of the parallel diffusion operator on the discrete level.

\section{Numerical Approach}

\subsection{Grid and Perpendicular Operators}

The problems of numerical grids constructed with straight field line coordinates can be seen in fig. 1. Since $q \rightarrow \infty$ at the X-point the contours of the straight poloidal angle are sucked into the X-point. Moreover, the flux surfaces are stretched apart close to the X-point and squeezed at the outboard midplane. On the contrary turbulent structures tend to be isotropic in planes perpendicular to the magnetic field. The structures are thus better represented on an isotropic cartesian grid independent from flux surfaces.

As a grid few poloidal planes are chosen as cuts at different toroidal angles $\varphi_{k}$ (see fig. 2a). Within these poloidal planes the cartesian isotropic grid is bounded by two extreme flux surfaces (see fig. 2b). The flute mode character $\left(k_{\|} \ll k_{\perp}\right)$ of the turbulent structures will be exploited by choosing a low resolution in the toroidal direction and a field line following discretisation of parallel operators. Due to the independence from flux surfaces - except for the limiting flux surfaces - a separatrix can be treated easily. This approach is similar to the one described in [5].

\footnotetext{
* Corresponding author E-mail: Andreas.Stegmeir@ipp.mpg.de, Phone: +498932992210, Fax: +49 8932992580
} 


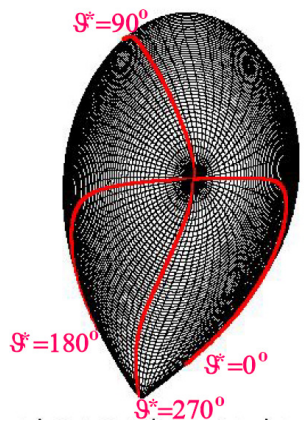

Fig. 1 Numerical grid constructed with straight field line coordinates [4].
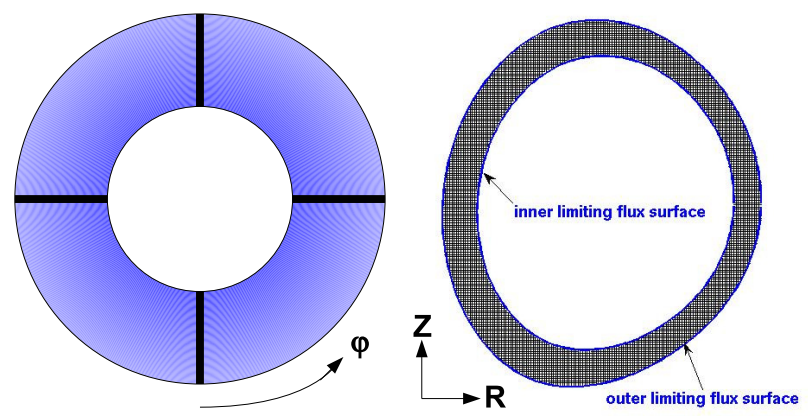

Fig. 2 Numerical grid. a: View of tokamak from above with cuts at 4 different toroidal angles are taken. b: Within each poidal plane the grid is Cartesian isotropic bounded by two extrem flux surfaces.

Due to the strong guiding toroidal field, operators which describe dynamics perpendicular to magnetic field lines (e.g. $\nabla_{\perp}^{2}$ ) can be approximated with a stencil within a poloidal plane. Derivatives with respect to $\varphi$ can be neglected against derivatives in $R$ or $Z$ direction $\left(k_{R}, k_{Z} \gg k_{\varphi}\right)$. Derivatives with respect to $R$ and $Z$ can be discretized with standard second order finite difference methods (e.g. [6]).

\subsection{Parallel Gradient}

Parallel operators connect information between neighbouring poloidal planes. Due to the low resolution in the toroidal direction also the magnetic field line structure between the poloidal planes must be accounted for at the discretisation of parallel operators. For each grid point $(i, j, k)$ a field line tracing along the magnetic field line is performed. The penetration points $R_{i j k}^{\alpha, \beta}, Z_{i j k}^{\alpha, \beta}$ at the neighbouring poloidal planes and lengths along the field lines $\Delta s_{i j k}^{\alpha, \beta}$ are computed with a field line tracing procedure, where $\alpha / \beta$ denotes co/counter-direction of the magnetic field line (see fig 3).

Since the penetration points in general do not coincide with a grid point the value of the considered quantity at the penetration point is obtained by interpolation. Results for a bilinear interpolation (bilinear) involving 4 points and a 3rd order polynomial interpolation (polynomial3) involving 16 points will be shown in sec. 3 . The parallel gradients between the poloidal plane can then be approximated with the interpolated values by a finite difference:

$$
\nabla_{\|}^{\alpha} u_{i j k}=\frac{u\left(R_{i j k}^{\alpha}, Z_{i j k}^{\alpha}\right)-u_{i j k}}{\Delta s_{i j k}^{\alpha}}, \quad \nabla_{\|}^{\beta} u_{i j k}=\frac{u_{i j k}-u\left(R_{i j k}^{\beta}, Z_{i j k}^{\beta}\right)}{\Delta s_{i j k}^{\beta}} .
$$

The parallel gradient at the considered grid point could be obtained via a further interpolation along the magnetic field line with these two values to the grid point, but this shall not be discussed here (see also [5]). The bilinear/polynomial3 interpolation schemes are linear in the grid values which allows to write the parallel gradient as linear transformation $\left(u_{i j k} \rightarrow \mathbf{u}, \nabla_{\|}^{\alpha, \beta} u_{i j k} \rightarrow \mathbf{q}^{\alpha, \beta}, \mathbf{q}^{\alpha, \beta}=\mathbf{Q}^{\alpha, \beta} \mathbf{u}\right)$.

\subsection{Parallel Diffusion}

\subsubsection{Naive Discretisation}

By simply neglecting $\nabla \cdot \mathbf{b}$, the parallel diffusion operator could be approximated as twice application of the parallel gradient. A finite difference of the parallel gradient can then be applied further:

$$
\mathcal{D} u:=\nabla \cdot\left[\left(\nabla_{\|} u\right) \mathbf{b}\right] \approx \nabla_{\|}^{2} u \rightarrow \frac{\nabla_{\|}^{\alpha} u_{i j k}-\nabla_{\|}^{\beta} u_{i j k}}{\left(\Delta s_{i j k}^{\alpha}+\Delta s_{i j k}^{\beta}\right) / 2} .
$$

However, we will see that this discretisation scheme has bad convergence properties and comparably high numerical decay rates. 


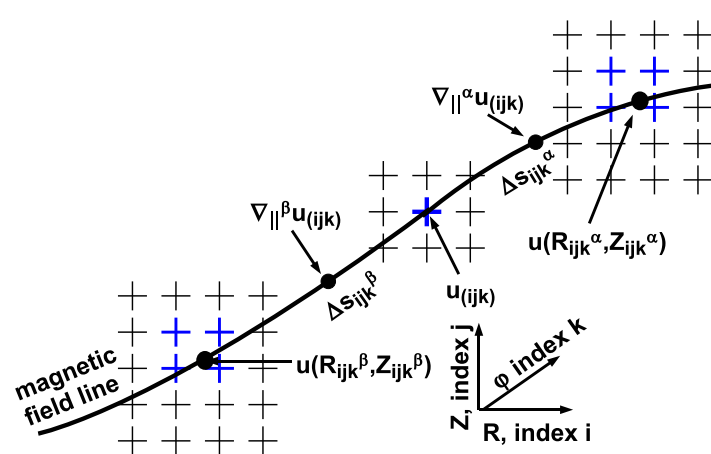

Fig. 3 Sketch of discretisation for parallel gradient. Blue points represent the points involved in the computation for the parallel gradient (bilinear stencil).

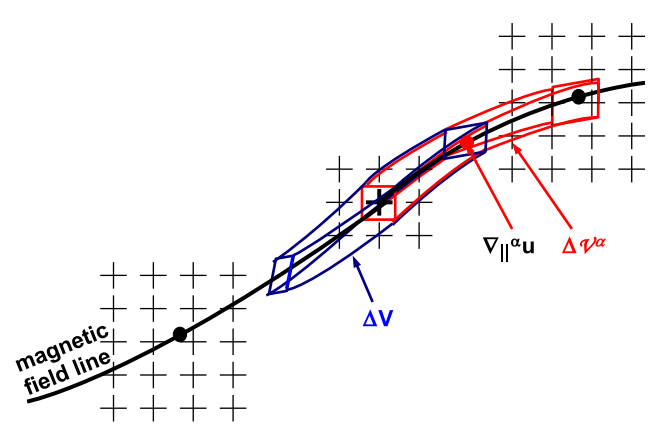

Fig. 4 Sketch for discretisation of scalar products for node grid quantities ( $N G$, blue) and flux quantities $(C V \alpha$, red $)$

\subsubsection{Support Operator Method}

The way to achieve a scheme with better convergence properties and a lower level of numerical damping was lead by the thought to maintain or mimic 'good' properties of the parallel diffsuion operator on the discrete level. The self-adjointness or the property that the parallel divergence is the negative adjoint of the parallel gradient is obvious:

$$
\langle v, \mathcal{D} u\rangle=\langle\mathcal{D} v, u\rangle, \quad \nabla \cdot[\mathbf{b} \circ]=-\nabla_{\|}^{\dagger}
$$

for arbitrary functions $u, v$, which are zero at the boundaries. The brackets denote integration over the whole volume and $\mathcal{D}$ is the parallel diffusion operator as defined in eq. 2.

With the method of support operatos [7] a finite difference scheme is constructed, which mimics the relations 3 on the discrete level. This method was also applied in [8]. The parallel gradient is chosen as the prime operator and is discretised according to eq. 1. In the following we present the discretisation of the parallel diffusion operator with the $\alpha$-discretisation $\left(\nabla_{\alpha}^{\|}\right)$. The $\beta$-discretisation can be obtained analogously.

Scalar quantities $u, v$ are represented on the node grid $(N G)$, fluxes $q^{\alpha}, p^{\alpha}$ are represented with cell volumes between the poloidal planes $(C V \alpha)$. Thus the discrete parallel gradient maps from $(N G)$ to $(C V \alpha)$. The scalar product at the discrete level is mimiced for scalar quantities and for fluxes according to:

$$
\langle v, u\rangle_{N G}=\sum_{l} v_{l} u_{l} \Delta V_{l}, \quad\left\langle p^{\alpha}, q^{\alpha}\right\rangle_{C V \alpha}=\sum_{m} p_{m}^{\alpha} q_{m}^{\alpha} \Delta \mathcal{V}_{m}^{\alpha}
$$

where $\Delta V_{l}$ and $\Delta \mathcal{V}_{m}^{\alpha}$ are flux box volumes around the considered magnetic field line. The definition and construction of these volumes can be deduced from fig. 4 and is also described at the end of this section. Both discretisations of the scalar products mimic an integration over the same whole simulation domain. From the discretisation of the parallel gradient, the discretised scalar products and the requirements of eqs. 3 follows the discretized parallel divergence as the derived operator. The discretised parallel diffusion operator is:

$$
\mathcal{D} u \rightarrow\left(\mathbf{D}^{\alpha} \mathbf{u}\right)_{l}=-\sum_{m, j} \mathbf{Q}_{l m}^{\alpha} \mathbf{Q}_{m j}^{\alpha} \frac{\Delta \mathcal{V}_{m}^{\alpha}}{\Delta V_{l}} u_{j}
$$

There is a last subtle point. At the beginning we chose for the parallel gradient the $\alpha$ discretisation. The same derivation with the $\beta$ discretisation will end up in a slightly different scheme in general. Both ways are consistent and converge towards the continuous parallel diffusion operator. However, on the discrete level a preservation of the toroidal symmetry of the operator $(\varphi \rightarrow-\varphi)$ is desirable and achieved by simply taking the average of both schemes $\left.\left(\mathbf{D}=\left(\mathbf{D}^{\alpha}+\mathbf{D}^{\beta}\right) / 2\right)\right)$.

Based on the frozen flux theorem of ideal MHD, the flux box volumes can be computed during the field line 

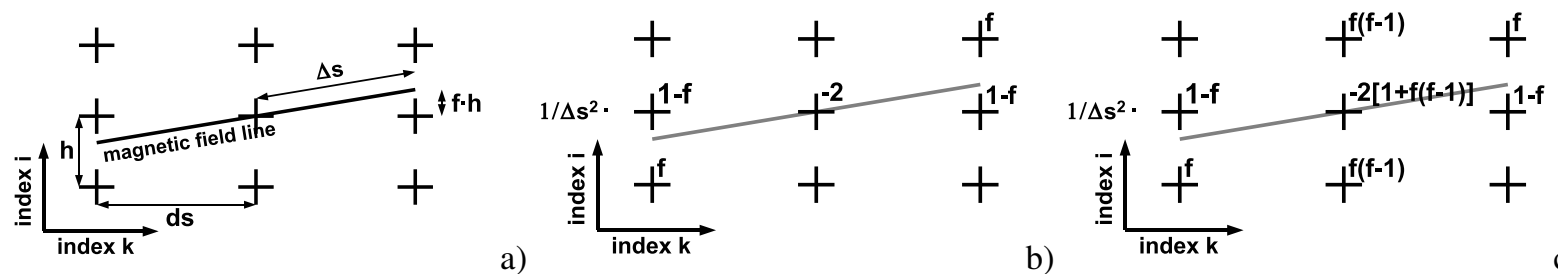

Fig. 5 a: Sketch of minimum nontrivial problem. b: Stencil for inner grid point with naive scheme. c: Stencil with support scheme.

tracing procedure according to:

$$
\Delta V(\varphi)=\int_{0}^{\varphi} \frac{B_{0}^{t o r} A_{0}}{B^{\text {tor }}(\mathbf{s})} \frac{\mathbf{e}_{\varphi}}{\left|\mathbf{e}_{\varphi}\right|} \cdot d \mathbf{s}(\varphi),
$$

where $\mathbf{s}$ is the path along the magnetic field line, $B_{0}^{\text {tor }}$ is the toroidal field strength and $A_{0}=d R \cdot d Z$ the area (spanned perpendicular to $\mathbf{e}_{\varphi}$ ) at the start of the path.

\subsection{Minimum nontrivial problem (2D)}

Expressions 2 (naive) and 5 (support) are general expressions of the discrete parallel diffusion operator, which are valid for quite arbitrary geometries and interpolation techniques. In order to shed some more light on these expressions, a minimum nontrivial 2D model problem is considered here. It consists of a 9 point regular grid illustrated in fig. 5a. The magnetic field is uniform but slightly inclined with respect to the grid expressed with the factor $f$. Since we are only interested in the discretisation of an inner grid point (here only point $(2,2)$ ) effects from outside the domain are not important ( $u=0$ at exterior ghost points). Using a linear interpolation to compute the value of the considered quantity at the penetration points the discrete parallel gradient in matrix form is $\left(\mathbf{u}=\left(u_{1,1}, u_{2,1}, u_{3,1}, u_{1,2}, \ldots u_{3,3}\right)^{T}\right)$ :

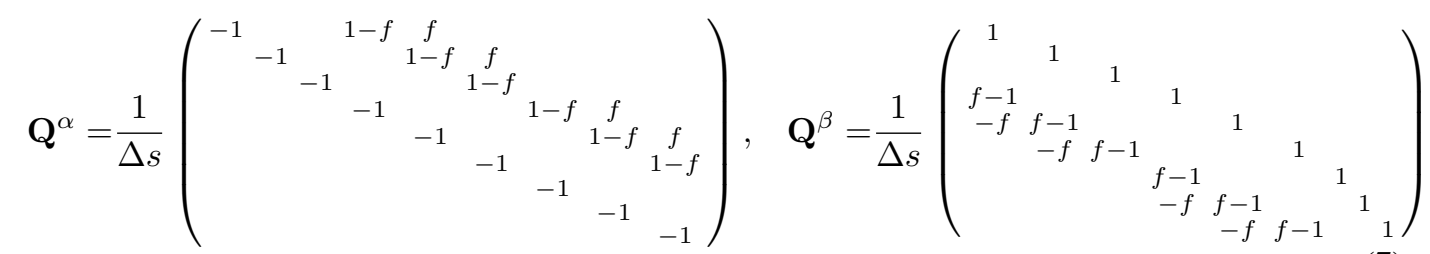

In this simplified case the discrete volumes are all equal $\Delta V_{i k}=\Delta \mathcal{V}_{i k}^{\alpha, \beta}=h \cdot d s$. The stencil of the discrete parallel diffusion operator for an inner grid point is shown in fig. 5b,c. If the penetration points coincide with grid points $(f=0$ or $f=1)$, the standard second order finite difference expression is obtained for both schemes. The stencil of the naive scheme only involves the considered grid point itself and grid points at neighbouring poloidal planes $(k-1$ and $k+1)$ which contribute to the interpolation. The support scheme involves additionally also grid points which are located in the same plane as the considered grid point.

\section{Test of Numerical Scheme}

\subsection{Convergence}

The convergence properties of the parallel diffusion operator is investigated with a parallel diffusion equation. For the case of an axial periodic cylinder $\left(x, y, z\right.$, with $z \in[0,2 \pi]$ periodic, $\rho=\sqrt{x^{2}+y^{2}}$, safety factor $q=q(\rho)$ ) an analytic solution is available. Let the initial state be characterised by a poloidal and toroidal mode number $(m, n)$ and an arbitrary radial structure $f(\rho)$. The analytic solution is given by:

$$
u_{a n}(t)=f(\rho) \exp (-\gamma(\rho) t) \quad \text { with } \quad \gamma(\rho)=\frac{1}{1+\rho^{2} / q(\rho)^{2}}\left(\frac{m}{q(\rho)}+n\right)^{2} .
$$



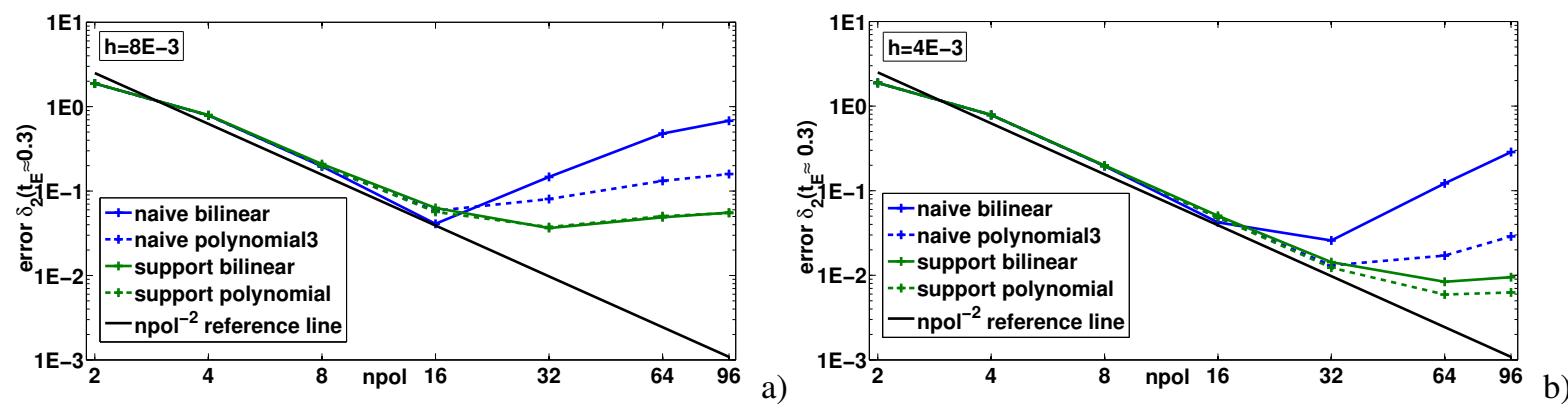

Fig. 6 Numerical error of the parallel diffusion equation in dependence on the number of poloidal planes (toroidal resolution) for two different resolutions within the poloidal plane (a: low $h=8 E-3$, b: high $h=4 E-3$
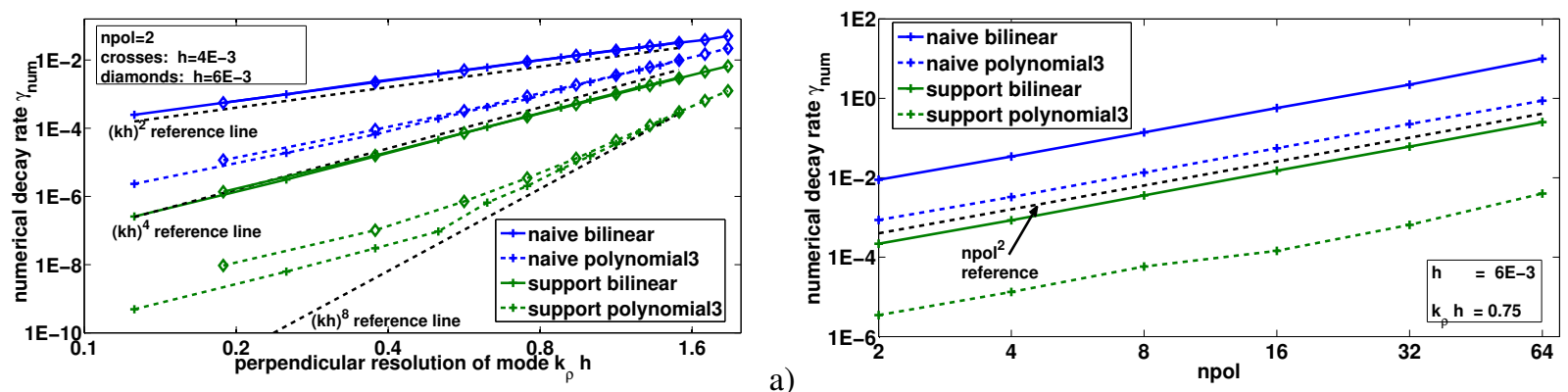

a)

Fig. 7 Numerical decay rate a: in dependence on radial mode resolution $k_{\rho} h$ for fixed number of poloidal planes $(n p o l=2)$, b: in dependence on toroidal resolution for fixed poloidal resolution $h=6 E-3$

For the test reported here a constant safety factor of $q(\rho)=3.4$ was choosen. The flux shell is limited by two flux surfaces with $\rho_{\min }=0.1$ and $\rho_{\max }=0.2$. The initial state is given by a $(m=3, n=1)$ mode with a radial structure of $f(\rho)=\sin \left(\pi\left(\rho-\rho_{\min }\right) /\left(\rho_{\max }-\rho_{\min }\right)\right)$.

In fig. 6 the error $\delta_{2}=\left\|u_{a n}-u_{\text {num }}\right\|_{2} /\left\|u_{a n}\right\|_{2}$ at the time $t_{e}=\gamma^{-1}\left(\left(\rho_{\max }+\rho_{\min }\right) / 2\right) \approx 0.3$ is plotted for the different schemes in dependence on the number of poloidal planes $n p o l$, i.e. the toroidal resolution. The error is plotted for two different resolutions within the poloidal plane. At low toroidal resolution the error decays like $n \mathrm{pol}^{-2}$ for all schemes, which shows the second order accuracy. At a higher number of poloidal planes the error due to the interpolation begins to dominate and increases with npol. The convergence along the $n_{p o l}{ }^{-2}$ line extends for the support schemes to a higher toroidal resolution than for the naive scheme.

\subsection{Numerical Decay}

To investigate the scheme with respect to its numerical decay rate the same setup as in sec. 3.1 is chosen with $q=3$. The initial state is given by $u(t=0)=\sin \left(\pi r \frac{\rho-\rho_{\min }}{\rho_{\max }-\rho_{\min }}\right)$, where $r$ is a radial mode number. This initial state has a vanishing parallel gradient and the damping of this mode is a purely numerical effect. In fig. 7a the numerical decay rate $\gamma_{n u m}$ is plotted in dependence on the radial resolution of the mode and in fig. $7 \mathrm{~b}$ in dependence on the toroidal resolution for a fixed poloidal resolution. Let $p$ be the order of the error of the interpolation scheme. From the graphs can be deduced (except at high poloidal resolutions for the support scheme, where the error is dominated by effects close to the radial boundary):

$$
\begin{aligned}
\gamma_{n u m} & \propto C_{1} \operatorname{npol}^{2}\left(k_{\rho} h\right)^{p} \\
\gamma_{n u m} & \propto C_{2} \operatorname{npol}^{2}\left(k_{\rho} h\right)^{2 p}
\end{aligned}
$$

for naive scheme

for support scheme

where $C_{1}$ is larger then $C_{2}$. Additional tests showed that this result is independent on shear. The strong dependence of the numerical decay rate with $\left(k_{\rho} h\right)^{8}$ makes the support-polynomial3 scheme robust for already moderate resolutions. 

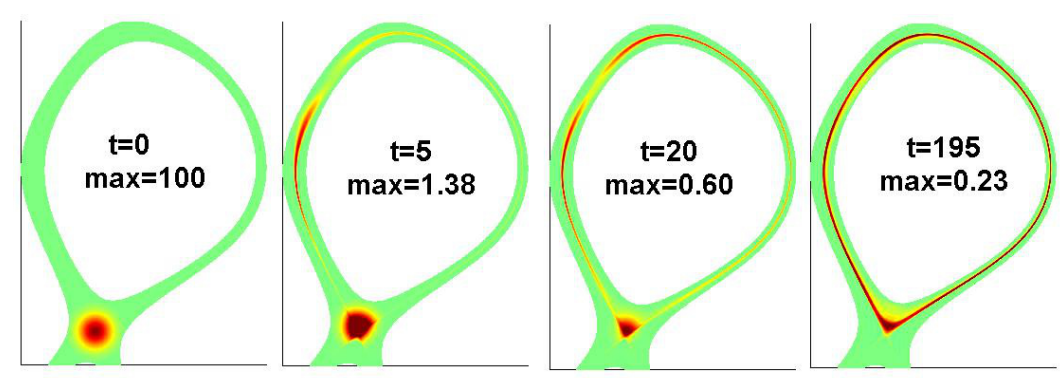

Fig. 8 Temporal evolution of a blob situated on the $\mathrm{X}$-point at $\varphi=0$. (npol $=48, h=5 E-$ $\left.4\left[R_{0}\right]\right)$

\subsection{Application to Separatrix}

To show the applicability of the numerical scheme to equilibria where an X-point is present the parallel diffusion equation is solved with Dirichlet boundary conditions $(u=0)$ at the intersection points of the magnetic field lines with the plates. The initial state is a toroidally and poloidally limited gaussian blob at the X-point. The temporal evolution of the system is shown in fig. 8. In the open field line region the blob is diffused away to the plates. In the closed field line region the quantity equilibrates along the magnetic field lines and the flux surfaces become apparent.

\section{Conclusions and Outlook}

A numerical approach suitable for X-point geometries is presented which works with a flux surface independent grid. A discretisation scheme for the parallel diffusion operator which maintains the self-adjointness on the discrete level is constructed. The numerical decay rate for this scheme is at very low levels already for moderate resolutions.

The next step is to apply these methods to a simple turbulence model (e.g. Hasegawa Wakatani [9]) and investigate its dynamics close to the $\mathrm{X}$-point.

\section{References}

[1] A. Loarte et al., Nucl. Fusion 47, S203 (2007).

[2] P. Ricci et al., Plasma Phys. Control. Fusion 54, 124047 (2012).

[3] W.D. D'haeseleer, W. N. G. Hitchon, J.D. Callen and J. L. Shohet, Flux Coordinates and Magnetic Field Structure (Springer, Berlin, 1990).

[4] E. Strumberger, THE VMEC CODE: principle and applications, presentation at IPP Garching (2012)

[5] F. Hariri and M. Ottaviani, Comp. Phys Comm., in press (2013).

[6] W. F. Ames, Numerical Methods for Partial Differential Equations, 3rd edition (AP, Boston, 1992), p. 18

[7] M. Shaskov, Conservative Finite-difference Methods on General Grids, (CRC, Boca Raton, 1996)

[8] S. Günter et al., Journ. Comp. Phys. 209, 354 (2005)

[9] A. Hasegawa and M. Wakatani, Phys. Rev. Lett. 50, 682 (1983) 